\title{
Perceptions of patients and providers on myocardial perfusion imaging for asymptomatic patients, choosing wisely, and professional liability
}

Kristopher P. Kline', Leslee Shaw², Rebecca J. Beyth³, Jared Plumb4 ${ }^{4}$, Linda Nguyen ${ }^{5}$, Tianyao Huo ${ }^{6}$ and David E. Winchester ${ }^{4,6 *}$

\begin{abstract}
Background: Despite efforts by professional societies to reduce low value care, many reports indicate that unnecessary tests, such as nuclear myocardial perfusion imaging (MPI), are commonly used in contemporary practice. The degree to which lack of awareness and professional liability concerns drive these behaviors warrants further study. We sought to investigate patient and provider perceptions about MPI in asymptomatic patients, the Choosing Wisely (CW) campaign, and professional liability concerns.

Methods: We administered an anonymous, paper-based survey with both discrete and open-response queries to subjects in multiple outpatient settings at our facilities. The survey was completed by 456 respondents including 342 patients and 114 physicians and advanced practice providers between May and August 2014. Our outcome was to compare patient and provider perceptions about MPI in asymptomatic patients and related factors.

Results: Patients were more likely than providers to report that MPI was justified for asymptomatic patients (e.g. asymptomatic with family history of heart disease $75 \%$ versus $9.2 \%, p<0.0001$ ). In free responses to the question "What would be an inappropriate reason for MPI?" many responses echoed the goals of CW (for example, "If you don't have symptoms", "If the test is too risky", "For screening or in asymptomatic patients"). A minority of providers were aware of CW while even fewer patients were aware $(37.2 \%$ versus $2.7 \%, p<0.0001)$. Over one third of providers (38.9\%) admitted to ordering MPI out of concern for professional liability including $48.3 \%$ of VA affiliated providers.

Conclusions: While some patients and providers are aware of the low value of MPI in patients without symptoms, others are enthusiastic to use it for a variety of scenarios. Concerns about professional liability likely contribute, even in the VA setting. Awareness of the Choosing Wisely campaign is low in both groups.
\end{abstract}

Keywords: Myocardial perfusion imaging, Appropriate use criteria, Choosing wisely, Medical liability

\footnotetext{
* Correspondence: david.winchester@medicine.ufl.edu

${ }^{4}$ Department of Medicine, College of Medicine, Division of Cardiovascular

Medicine, University of Florida, 1600 SW Archer Road, PO Box 100277,

Gainesville, FL 32610-0277, USA

${ }^{6}$ Cardiology Section, Medical Service, Malcom Randall VA Medical Center,

Gainesville, FL, USA

Full list of author information is available at the end of the article
} 


\section{Background}

Waste in medical spending is a widespread problem, estimated to be $30 \%$ of all health spending and equivalent to 750 billion US dollars annually $[1,2]$. A variety of initiatives have been created by the medical profession to curb unnecessary spending, such as the Choosing Wisely (CW) campaign. This initiative was started by the American Board of Internal Medicine Foundation (ABIM) and asked medical societies to identify five things that patients and physicians should question. Lists created by multiple specialty societies cite overuse of nuclear cardiac stress testing or myocardial perfusion imaging (MPI) [3]. High rates of unnecessary MPI testing places burden on the medical system while exposing patients to unwarranted risks. In another initiative to reduce unnecessary and wasteful testing, professional societies have also developed Appropriate Use Criteria (AUC) to assist doctors in deciding which patients may benefit from MPI in common clinical scenarios [4].

These efforts are laudable, but their acceptance and implementation by front line providers in clinical care is unknown. Furthermore, the role of patients in these processes is limited, or nonexistent; patients' reflections on appropriateness and awareness of waste and liability risks have not been studied. To investigate these issues, we conducted a survey of patients and healthcare providers asking a variety of questions related to these issues. We hypothesized that patients would be more likely than providers to endorse the use of MPI in the absence of symptoms.

\section{Methods}

\section{Survey questionnaire}

We developed and administered an anonymous survey to patients who have had MPI or had one ordered for them in primary care clinics, cardiology clinics, and nuclear medicine laboratories at both our academic medical center and affiliated Veterans Affairs (VA) medical center. We developed another survey with a parallel structure and administered it to healthcare providers, sampled from physicians and advanced providers (nurse practitioners and physician assistants) within primary care, cardiology, and hospital medicine. Providers were from within the same academic medical center. Its affiliated VA medical center, and private practice. When appropriate, identical questions were asked of both patients and providers.

Respondents provided demographic information and answered a wide variety of questions relating to the CW campaign, professional liability, unnecessary MPI, and wasted medical spending. Patients and providers were asked questions to gauge understanding on risks versus benefits of MPI testing. Survey questions were in a variety of formats including yes/no, scale responses, multiple choice, and narrative/free response. (For sample question please refer to Fig. 1; full questionnaire available as Additional files 1 and 2) Patient and provider attitudes towards AUC for MPI from this survey have been previously reported; these survey questions and those for asymptomatic testing were developed from previous studies on inappropriate MPI. [5-7] Survey questions for $\mathrm{CW}$, professional liability and wasted medical spending were developed by authors to investigate potential contributing factors.

\section{Survey administration}

The study was approved by our Institutional Review Board. Surveys were administered in paper form between May and August 2014. Informed consent was obtained, however the requirement for written documentation of informed consent was waived in order to maintain anonymity for all participants. Patient participants were recruited by study personnel (KK, JP, LN, DW) at patient check-in for appointments at a variety of clinical locations as noted above. Blank forms were also made available at appointment check-in to be completed voluntarily. Providers within our academic medical center and affiliated VA medical center and recruited through staff meetings; providers from private practice were recruited at statewide professional meetings and

\footnotetext{
Patient Questions:

Have you ever specifically told your doctor you wanted a specific test done such as a blood test, stress test, or X ray? Yes No

Is yes, how does your doctor usually respond when you ask for a test to be done? Orders the test Discusses the test with you before deciding Refuses to order the test

Provider Questions:

How often do patients ask for a test or procedure that you think is unnecessary?

Every day Almost every day Several times a week About once a week At least once a month Less often

When patients ask for an unnecessary test or procedure, how often do you talk to them about why they should not have the test or procedure? Always Almost always Frequently Not too often Rarely or never
}

Fig. 1 Sample questions showing parallel structure as reported to patient respondents versus provider respondents 
through personal interactions. Because of this methodology, and because regulatory oversight authorities did not permit formal tracking of those who were approached but did not elect to participate, an accurate denominator to calculate the percent responding to the survey cannot be provided. Results from completed surveys were entered into the secure, web-based application Research Electronic Data Capture (REDCap) database (authors JP, KK, and LN) [8].

\section{Statistical analysis}

Baseline characteristics and survey responses were compared by ANOVA, Chi-square test, Fisher's exact test, or Mantel-Haenszel test as appropriate for continuous, categorical, or ordinal variables as appropriate. Statistical calculations were performed on SAS version 9.4 (SAS Institute, Cary NC).

\section{Results}

The survey was completed by 456 respondents, 342 patients and 114 providers. Three hundred fourty two patient respondents were distributed equally between cardiology $(32.2 \%)$, primary care $(36.0 \%)$, and nuclear medicine $(31.9 \%)$ clinics; $55.6 \%$ were Veterans. (Table 1) Patient respondents were predominantly male (68\%), and finished at least some college education (67.5\%).
Providers were from academic practice (61\%), VA practice $(27 \%)$, or private practice $(10 \%)$. The majority were physicians (87\%) followed by advanced care providers (13\%). Primary specialty was cardiology (35.1\%), primary care $(37.7 \%)$, hospital medicine $(22.8 \%)$, and other (4.4\%).

\section{MPI in asymptomatic patients}

Patients and providers were asked to consider a patient without symptoms and in what situations it would be appropriate to perform MPI, results are shown in Table 2. $75 \%(n=238)$ of patients believed a family history of coronary artery disease warranted an MPI compared to just $9.2 \%(n=10)$ of providers. $33 \%(n=96)$ of patients considered having erectile dysfunction an indication for an MPI, while just $3.6 \%(n=4)$ of providers agreed with this statement. Only $1(0.9 \%)$ provider thought annual evaluation with MPI for asymptomatic patients was indicated, compared to almost a quarter $(23 \%, n=71)$ of patients.

\section{Unnecessary testing}

We asked respondents to detail their experience with patient driven requests for specific testing. When we asked patients if they have ever specifically asked their provider to order a test, $40 \%$ responded that they had.

Table 1 Patient and provider baseline characteristics

\begin{tabular}{|c|c|c|c|}
\hline & Total & & Total \\
\hline Patients & $n=342$ & Providers & $n=114$ \\
\hline Gender (male) & $232(68 \%)$ & Professional Training & \\
\hline Age & $61 \pm 14$ & MD/DO & 99 (87\%) \\
\hline Prior myocardial infarction & $62(18 \%)$ & ARNP/PA & $15(13 \%)$ \\
\hline Prior PCl & $56(16 \%)$ & & \\
\hline Prior CABG & $48(14 \%)$ & Level of Experience & \\
\hline Diabetes & $126(37 \%)$ & In training & $48(42 \%)$ \\
\hline High blood pressure & $236(69 \%)$ & $<5$ years & $20(17.5 \%)$ \\
\hline High cholesterol & $180(53 \%)$ & $5-15$ years & $23(20 \%)$ \\
\hline Education (all responses) & & $>15$ years & $23(20 \%)$ \\
\hline Did not finish high school & $19(6 \%)$ & & \\
\hline Finished high school & $87(26 \%)$ & Primary specialty & \\
\hline Some college classes & $118(35 \%)$ & Primary Care & $43(38 \%)$ \\
\hline Graduated college & $69(20 \%)$ & Hospital Medicine & $26(23 \%)$ \\
\hline \multirow[t]{7}{*}{ Graduate or professional school } & $44(13 \%)$ & Cardiology & $40(35 \%)$ \\
\hline & & Other & $5(4 \%)$ \\
\hline & & Primary workplace & \\
\hline & & Academic & 71 (62\%) \\
\hline & & VA & $31(27 \%)$ \\
\hline & & Private & $9(7 \%)$ \\
\hline & & Other academic & $3(3 \%)$ \\
\hline
\end{tabular}


Table 2 Patient and provider opinions on what justifies an MPI in asymptomatic patients

\begin{tabular}{llllll}
\hline Which of the following justify an MPI? & Patient & Provider & $p$ value & OR & $95 \%$ Cl \\
\hline Diabetes mellitus & $102(35 \%)$ & $28(25.7 \%)$ & 0.093 & 1.55 & $0.92-2.61$ \\
ECG changes & $263(86 \%)$ & $63(57.3 \%)$ & $<0.0001$ & 4.56 & $2.70-7.73$ \\
Family history of CAD & $238(75 \%)$ & $10(9.2 \%)$ & $<0.0001$ & 30.21 & $14.43-64.95$ \\
Erectile dysfunction & $96(33 \%)$ & $4(3.6 \%)$ & $<0.0001$ & 12.91 & 7.73 \\
High risk of heart disease & $272(86 \%)$ & $48(44.4 \%)$ & $<0.0001$ & 32.79 & $4.42-42.47$ \\
Annual evaluation & $71(23 \%)$ & $1(0.9 \%)$ & $<0.0001$ & 10.08 & $2.97-41.09$ \\
Preoperative evaluation for low-risk surgery & $67(22 \%)$ & $3(2.7 \%)$ & $<0.0001$ & 22.94 & $12.09-43.76$ \\
Prior history of Ml 1 year prior & $246(80 \%)$ & $16(14.5 \%)$ & $<0.0001$ & $<0.97$ \\
Prior history of stents 1 year prior & $209(69 \%)$ & $15(13.6 \%)$ & $<001$ & 13.93 & $7.4-26.51$ \\
\hline
\end{tabular}

twenty five reported that the provider orders the test without any discussion or inquiry as to why the patient wanted the study. The most common reasons patients reported requesting a test were family history of disease (23\%), risk factors $(16 \%)$, or at the recommendation of a family member (4\%). Only 3 respondents (1\%) requested a study because they heard about it on television or the internet. Among providers, 13.3\% reported that patients requested unnecessary tests every day in their practice; $23.9 \%$ indicated this occurs almost daily. Among providers receiving frequent requests for unnecessary testing, $44.3 \%$ were academic, $33 \%$ private and $17.2 \%$ VA $(p=0.034)$.

Both providers and patients were asked to reflect on reasons why testing might be inappropriate. (Table 3) Many of the provider responses could be matched to an inappropriate MPI indication while some responses, such as liability concern and patient/family demand is not specifically noted in the AUC. Providers indicated that $31 \%$ of them have ordered a MPI only because the patient demanded it. Patient and provider narrative responses by rank and their correlation to published AUC are shown in Table 3.

Table 3 Patient and provider narrative responses by rank and their correlation to published AUC

\begin{tabular}{|c|c|c|}
\hline Patients: Inappropriate Reasons for MPI $(n=149)$ & 2009 AUC & Numbe \\
\hline If the test is too risky & N/A & 11 \\
\hline If you do not have symptoms & \#7-11 & 10 \\
\hline If the test is not necessary & N/A & 10 \\
\hline If the radiation risk is high & N/A & 6 \\
\hline The doctor should decide & N/A & 6 \\
\hline If the cost is too high & N/A & 1 \\
\hline False positive testing & N/A & 0 \\
\hline Providers: Inappropriate reasons for MPI & 2009 AUC & Numbe \\
\hline Asymptomatic & \#7-11 & 26 \\
\hline Patient or family demand & N/A & 16 \\
\hline Concern about professional liability & N/A & 12 \\
\hline Screening in low risk patients & \#7 & 9 \\
\hline Annual MPI & \#45-56, \#66-70 & 8 \\
\hline Preoperative risk assessment & $\# 71$ & 8 \\
\hline Symptoms with a normal ECG and can exercise & $\# 1$ & 8 \\
\hline Low risk chest pain & $\# 1-2$ & 6 \\
\hline Acute coronary syndrome & $\# 10$ & 6 \\
\hline Repeat test, no change in symptoms & $\# 45-56, \# 66-70$ & 5 \\
\hline Financial gain & N/A & 4 \\
\hline Risk factors/Family History/Abnormal ECG alone & N/A & 4 \\
\hline Chest pain with high pretest likelihood & \#5-6 & 3 \\
\hline
\end{tabular}




\section{Choosing wisely}

$37.2 \%(n=42)$ of providers had heard of the CW campaign. Of the providers who were aware of $\mathrm{CW}$ a greater percentage were academic (48.6\%) compared to private practice $(11.1 \%)$ or the VA $(20.7 \%)(p=0.008)$. Proportionally more providers in training had heard of CW (44\%) compared to providers $<5$ years from training (19.5\%), 5-15 years from training (14.6\%) or $>15$ years from training $(9 \%)$ though this was not statistically significant $(p=0.59)$. Patients were less likely than providers to be aware of the CW campaign (2.7\% versus $37.2 \%, p<0.0001)$. In narrative responses, providers accurately described goals of the CW campaign, including appropriate testing recommendations, reduced waste spending, professional society based guidelines, and encouragement of shared decision making. Of the less than $3 \%$ of patients who had heard of the campaign, no narrative responses were reported describing the campaign's goals.

\section{Liability risks}

The majority of providers (65.2\%) felt their employment provided good or excellent professional liability protection; this proportion was significantly lower for private practice providers $(33.3 \%)$ compared to university affiliated $(73.9 \%)$ and VA providers $(55.2 \%)(p=0.027)$. Providers remain concerned about professional liability with $15.9 \%$ of respondents stating they were very concerned, $36.3 \%$ concerned, and $35.4 \%$ being slightly concerned. Only $12.4 \%$ reported being not concerned over professional liability. More than a third (38.9\%) of providers admitted that they had ordered a MPI in the past strictly out of concern for liability risk if they failed to do so.

\section{Waste and cost}

We created questions based on the cost of MPI, published estimates of the total cost (in US dollars), and the percentage of US healthcare spending that is wasted to determine if patients and providers had accurate assessments of these facts. Compared to patients, providers more frequently identified the correct total cost $(20.9 \%$ versus $10.4 \%, p<0.0001)$ and percentage of costs $(77.5 \%$ versus $39.3 \%, p<0.0001$ ) of wasteful spending. Over half of providers $(53.7 \%)$ and patients $(60 \%)$ correctly estimated the cost of a nuclear stress test to be $>\$ 1000$.

\section{Radiation and cancer risks}

In addressing risks of MPI, we asked questions about radiation exposure and understandings about radiation sources and risk. Two-thirds of patients correctly recognized that CT scans (65\%) and nuclear stress tests (67\%) were sources of radiation while $67 \%$ reported microwaves and $46 \%$ reported televisions were radiation sources. When asked to identify which entity had the most radiation exposure the top results were CT scans (33\%), nuclear stress tests (32\%) followed by microwaves $(18 \%)$. Close to half $(42 \%)$ of patient respondents reported there was no increased risk of cancer after getting MPI.

\section{Discussion}

We performed a large survey of both patients and providers taken predominantly from a single academic health center and affiliated VA hospital system to gauge opinions and attitudes about MPI, appropriateness, and a variety of related topics. The results of the survey indicate areas for improvement in understanding about MPI and provide some striking observations about unnecessary testing and professional liability.

Patient respondents to our survey were more likely to report that MPI was warranted in a variety of clinical scenarios for asymptomatic patients. This is in accord with a recent comprehensive systematic review that showed patients tend towards overestimating benefits while underestimating harm regardless of the intervention [9]. Patients are also poorly informed by their providers on the risks of over-diagnosis and overtreatment, as shown in a survey which noted only $10 \%$ of individuals were explained these risks when undergoing cancer screenings [10]. Contributing to information mismatch is the fact that providers are more likely to explain the pros of testing and treatments instead of the potential cons, and patients often do not feel involved in shared decision making [11-13]. Informed-decision making strategies via balanced discussions between patients and providers are key to improving these disparities.

In free-text responses about inappropriate tests, both providers and patients offered many accurate reasons why testing is unnecessary. Providers gave responses that matched a number of the AUC for MPI. Both also gave responses that indicated ongoing concerns and awareness of professional liability. Not surprisingly, our data contributes to the literature that physicians are less concerned about professional liability when they are employed by an entity with sovereign immunity $[14,15]$. We are concerned by the observation that over one third of providers openly admitted to ordering MPI specifically due to professional liability concerns. Similar findings were reported in a 2011 survey of primary care physicians showed that $76 \%$ ordered more aggressive testing due to liability concerns. [16] Another recent survey by an independent group for the American Board of Internal Medicine Foundation Choosing Wisely campaign reported that $52 \%$ of physicians felt the major reason for ordering unnecessary testing was professional liability concerns [17]. We observed among narrative responses from providers, liability concerns was the third most common "inappropriate" indication for ordering MPI behind asymptomatic patients and patient/family demand. 
In the same CW survey, $53 \%$ of ordering providers stated they would order an unnecessary test if a patient was insistent compared to $31 \%$ in our survey. Recent data suggest that "demanding" patients, those who go a clinical encounter with a specific request for a test or procedure, typically uncommon. Only $9 \%$ of encounters in outpatient oncology centers had such patients and that typically providers agreed with the patients' concerns [18]. Only $1 \%$ of these encounters were deemed to have a clinically inappropriate patient demand and that these were rarely fulfilled. While patient satisfaction appears to improve with increasing knowledge about their conditions, medications and procedures, some tests and procedures may be limited in their opportunity for patient involvement in decision-making [19]. The core of the CW campaign and patient-centered medicine is to respond to "demanding" patients with respectful understanding and communication about their concerns [20].

While a proportion of providers in our survey were aware of CW (37.2\%), we noted this was higher than national averages based on CW random polling data (21\%) [17]. We suspect this is secondary to our study being performed at an academic medical institution. Awareness of the campaign among patients was only $2.7 \%$ in our study as opposed to about $5 \%$ as a national average. This demonstrates a continued disparity between patient and provider knowledge of the campaign and low awareness of the campaign overall.

Respondents to our survey had modest awareness of waste in the US healthcare system and efforts to reduce it. Patients were less likely than providers to accurately estimate the amount of waste, both measured in US dollars and as a proportion of total spending. Polled physicians feel aware of costs for tests/treatments and think providers should adhere to guidelines that discourage marginally beneficial care [21]. An overwhelming 89\% agreed that doctors should play a bigger part in reducing unnecessary tests. A recent survey by Colla et al. showed nearly all physicians felt a responsibility to limit unnecessary testing, control costs, and adhere to guideline recommendations (96.8\%, 92.2\%, and 97.9\% respectively) [22]. Another important finding in our survey is an estimate of the proportion of "rarely appropriate" tests that would be acceptable. The Institute of Medicine has shown that over a quarter of the $\$ 750$ billion dollar annual cost of waste in health care is directly attributed to unnecessary services [2]. Currently, there is no agreement on what an acceptable rate of unnecessary services should be, but the most common response to this in our survey suggests a goal of less than $5 \%$ would be reasonable [7].

Patient responses also indicated a poor understanding of radiation exposure with $42 \%$ of respondents stating "no radiation exposure" associated with nuclear testing and $46 \%$ reported a "slight increase of exposure". This is similar to a recent survey showing that $85 \%$ of patients undergoing CT or cardiac SPECT imaging underestimated the amount of radiation and $88 \%$ were not worried about scan radiation [23]. Another survey noted only $3 \%$ of patients had been informed about radiation risks prior to their last imaging study [24]. This is a major concern when its estimated that greater than $10 \%$ of the ionizing radiation exposure in the U.S. can be directly attributed to nuclear stress testing [25]. Furthermore, providers are also often misinformed about radiation exposure [26]. A 2011 survey of American Society of Nuclear Cardiology members showed only 9\% could accurately estimate the radiation exposure of the most common nuclear stress test protocol [27]. Real time feedback has been proposed to assist with this problems as one recent study showed that displaying radiation exposure risks and imaging test costs at the time of ordering influenced providers on test appropriateness and helped facilitate physician-patient discussions [28]. More research is necessary to better guide informed decision-making for both patients and providers on radiation risks.

Our investigation has important limitations to acknowledge. The survey was conducted at a single site and may not be representative of broader populations. The methods used for population sampling may bias results towards a more favorable view of MPI. Responses were voluntary and rate of returned surveys were not tracked per Institutional Review Board regulations, leaving potential for bias due to absent responses. This is offset by the anonymous nature of the survey, especially regarding questions about professional liability and unnecessary testing. Survey responses by providers may not represent their actual clinical decision-making and ordering habits due to social desirability bias.

\section{Conclusion}

While efforts such as Choosing Wisely strive to improve communication and reduce low-value care, awareness and implementation of the campaign in the community remains low. Liability concerns and patient demand may still contribute to test overuse and ordering in rarely appropriate circumstances. Providers reported low rates of using AUC and had concerns about their purported benefits. Both patients and healthcare providers underestimate the costs of wasteful medical spending, patients to a greater degree. These findings suggest that further study and resources are needed to encourage patients and providers to adopt costconscious and appropriate medical testing.

\section{Additional files}

Additional file 1: Patient Survey. Description: Full version of questionnaire given to patients. (DOCX 25 kb) 
Additional file 2: Provider Survey. Description: Full version of questionnaire given to providers. (DOCX $27 \mathrm{~kb}$ )

\section{Abbreviations}

AUC: Appropriate Use Criteria; CW: Choosing Wisely; MPI: Myocardial perfusion imaging; VA: Veterans Affairs

\section{Acknowledgements}

Research reported in this publication was supported by the National Center for Advancing Translational Sciences of the National Institutes of Health under Award Number UL1TR001427. The content is solely the responsibility of the authors and does not necessarily represent the official views of the National Institutes of Health.

\section{Funding}

University of Florida Gatorade Endowment Fund. Gainesville, Florida. Discovery Pathways Program, Medical Student Research Program, Award Number 5T35HL007489-33. Florida Heart Research Institute. Malcom Randall VAMC. No funding sources had a role in the study design, data collection, analysis or interpretation of data. Funding sources did not participate in writing of the manuscirpt.

\section{Availability of data and materials}

The data generated or analysed during this study are available from the corresponding author on reasonable request.

\section{Authors' contributions}

All authors have contributed substantially to the study and manuscript including conception and design (all authors), acquisition of data (KK, JP, LN, $D W$ ), analysis and interpretation ( $L S, R B, T H, D W$ ), writing of the manuscript (KK, JP, DW), and critical revisions of manuscript (all authors). All authors have read and approved the final manuscript.

\section{Ethics approval and consent to participate}

Study was approved by University of Florida Institutional Review Board. All participants gave informed consent to participate.

\section{Consent for publication}

Not applicable.

\section{Competing interests}

The authors declare that they have no competing interests.

\section{Publisher's Note}

Springer Nature remains neutral with regard to jurisdictional claims in published maps and institutional affiliations.

\section{Author details \\ ${ }^{1}$ Department of Medicine, College of Medicine, University of Florida, Gainesville, FL, USA. ²Department of Medicine, Emory Clinical Cardiovascular Research Institute, Division of Cardiology, Emory University School of Medicine, Atlanta, GA, USA. ${ }^{3}$ Geriatric Research Education and Clinical Centers, Malcom Randall VA Medical Center, and University of Florida Department of Medicine, Gainesville, FL, USA. ${ }^{4}$ Department of Medicine College of Medicine, Division of Cardiovascular Medicine, University of Florida, 1600 SW Archer Road, PO Box 100277, Gainesville, FL 32610-0277, USA. ${ }^{5}$ College of Medicine, University of Florida, Gainesville, FL, USA. ${ }^{6}$ Cardiology Section, Medical Service, Malcom Randall VA Medical Center, Gainesville, FL, USA.}

Received: 5 April 2017 Accepted: 4 August 2017

Published online: 11 August 2017

\section{References}

1. Martin AB, Lassman D, Washington B, Catlin A. Growth in US health spending remained slow in 2010; health share of gross domestic product was unchanged from 2009. Health affairs (Project Hope). 2012;31(1):208-19.

2. Smith M, Saunders R, Stuckhardt L, McGinnis JM: Best care at lower cost: the path to continuously learning health care in America: National Academies Press; 2013.
3. American College of Cardiology: Five Things Physicians and Patients Should Question. http://www.choosingwisely.org/doctor-patient-lists/americancollege-of-cardiology/.

4. Hendel RC, Berman DS, Di Carli MF, Heidenreich PA, Henkin RE, Pellikka PA, Pohost GM, Williams KA. ACCF/ASNC/ACR/AHA/ASE/SCCT/SCMR/SNM 2009 appropriate use criteria for cardiac radionuclide imaging: a report of the American College of Cardiology Foundation appropriate use criteria task force, the American Society of Nuclear Cardiology, the American College of Radiology, the American Heart Association, the American Society of Echocardiography, the Society of Cardiovascular Computed Tomography, the Society for Cardiovascular Magnetic Resonance, and the Society of Nuclear Medicine. J Am Coll Cardiol. 2009;53(23):2201-29.

5. Winchester DE, Zhang P, Jadhav MP, Beyth RJ. A cohort study of myocardial perfusion imaging in veteran patients without symptoms: contributing factors and results of testing. Medicine. 2015;94(32):e1154.

6. Winchester DE, Hymas J, Meral R, Nguyen D, Dusaj R, Shaw L, Beyth RJ. Clinician-dependent variations in inappropriate use of myocardial perfusion imaging: training, specialty, and location. Journal of nuclear cardiology : official publication of the American Society of Nuclear Cardiology. 2014;21(3):598-604

7. Kline KP, Plumb J, Nguyen L, et al. Patient and Provider Attitudes on Appropriate Use Criteria for Myocardial Perfusion Imaging. JACC: Cardiovascular Imaging. 2017;10(7):824-25.

8. Harris PA, Taylor R, Thielke R, Payne J, Gonzalez N, Conde JG. Research electronic data capture (REDCap)-a metadata-driven methodology and workflow process for providing translational research informatics support. J Biomed Inform. 2009;42(2):377-81.

9. Hoffmann TC, Del Mar C. Patients' expectations of the benefits and harms of treatments, screening, and tests: a systematic review. JAMA Intern Med. 2015;175(2):274-86.

10. Wegwarth O, Gigerenzer G. Less is more: Overdiagnosis and overtreatment: evaluation of what physicians tell their patients about screening harms. JAMA Intern Med. 2013;173(22):2086-7.

11. Fowler FJ Jr, Gerstein BS, Barry MJ. How patient centered are medical decisions?: results of a national survey. JAMA Intern Med. 2013;173(13):1215-21.

12. Bauriedel MU. Just one more "noninvasive" test. JAMA Intern Med. 2014; 174(6):855.

13. Fagerlin A, Sepucha KR, Couper MP, Levin CA, Singer E, Zikmund-Fisher BJ. Patients' knowledge about 9 common health conditions: the DECISIONS survey. Med Decis Mak. 2010;30(5 Suppl):35s-52s.

14. Kini V, Weiner RB, McCarthy FH, Wiegers SE, Kirkpatrick JN. Association of Liability Concerns with decisions to order echocardiography and cardiac stress tests with imaging. J Am Soc Echocardiogr. 2016;

15. Zikmund-Fisher BJ, Kullgren JT, Fagerlin A, Klamerus ML, Bernstein SJ, Kerr EA. Perceived barriers to implementing individual choosing wisely(R) recommendations in two National Surveys of primary care providers. J Gen Intern Med. 2016;

16. Sirovich BE, Woloshin S, Schwartz LM. Too little? Too much? Primary care physicians' views on US health care: a brief report. Arch Intern Med. 2011; 171(17):1582-5.

17. Unnecessary Tests and Procedures in the Health Care System: What Physicians Say About the Problem, the Causes, and the Solutions.

18. Gogineni K, Shuman KL, Chinn D, Gabler NB, Emanuel EJ. Patient demands and requests for cancer tests and treatments. JAMA oncology. 2015;1(1):33-9.

19. Kebede S, Shihab HM, Berger ZD, Shah NG, Yeh HC, Brotman DJ. Patients' understanding of their hospitalizations and association with satisfaction. JAMA Intern Med. 2014;174(10):1698-700.

20. Back AL. The myth of the demanding patient. JAMA oncology. 2015;1(1):18-9.

21. Tilburt JC, Wynia MK, Sheeler RD, Thorsteinsdottir B, James KM, Egginton JS, Liebow M, Hurst S, Danis M, Goold SD. Views of US physicians about controlling health care costs. JAMA. 2013;310(4):380-8.

22. Colla CH, Kinsella EA, Morden NE, Meyers DJ, Rosenthal MB, Sequist TD. Physician perceptions of choosing wisely and drivers of overuse. Am J Manag Care. 2016;22(5):337-43.

23. Busey JM, Soine LA, Yager JR, Choi E, Shuman WP. Patient knowledge and understanding of radiation from diagnostic imaging. JAMA Intern Med. 2013;173(3):239-41.

24. Steele, J, Jones, A, Shoemaker, S. Patient Perception and Understanding of Radiation in Diagnostic Imaging. Radiological Society of North America. 2014 Scientific Assembly and Annual Meeting. Chicago IL. http://archive. rsna.org/2014/14018038.html. Accessed 9 Aug 2017. 
25. Einstein AJ. Effects of radiation exposure from cardiac imaging: how good are the data? J Am Coll Cardiol. 2012;59(6):553-65.

26. Rehani MM, Berris T. International Atomic Energy Agency study with referring physicians on patient radiation exposure and its tracking: a prospective survey using a web-based questionnaire. BMJ Open. 2012:2(5).

27. Einstein AJ, Tilkemeier P, Fazel R, Rakotoarivelo H, Shaw L. Radiation safety in nuclear cardiology-current knowledge and practice: results from the 2011 American Society of Nuclear Cardiology member survey. JAMA Intern Med. 2013;173(11):1021-3

28. Kruger JF, Chen AH, Rybkin A, Leeds K, Guzman D, Vittinghoff E, Goldman LE. Displaying radiation exposure and cost information at order entry for outpatient diagnostic imaging: a strategy to inform clinician ordering. BMJ quality \& safety. 2016;

Submit your next manuscript to BioMed Central and we will help you at every step:

- We accept pre-submission inquiries

- Our selector tool helps you to find the most relevant journal

- We provide round the clock customer support

- Convenient online submission

- Thorough peer review

- Inclusion in PubMed and all major indexing services

- Maximum visibility for your research

Submit your manuscript at www.biomedcentral.com/submit
Biomed Central 